\title{
Cloud formation from large-scale instabilities
}

\section{Woong-Tae Kim}

\author{
Department of Physics and Astronomy, FPRD, Seoul National University, Seoul 151-742, \\ Republic of Korea \\ email: wkim@astro.snu.ac.kr
}

\begin{abstract}
We discuss recent advances in cloud formation via gravitational instability under the action of self-gravity, magnetic fields, rotational shear, active stars, and/or stellar spiral arms. When shear is strong and the spiral arms are weak, applicable to flocculent galaxies at large, swing amplification exhibits nonlinear threshold behavior such that disks with a Toomre parameter $Q<Q_{c}$ experience gravitational runaway. For most realistic conditions, local models yield $Q_{c} \sim 1.4$, similar to the observed star formation thresholds. When shear is weak, on the other hand, as in galactic central parts or inside spiral arms, magneto-Jeans instability is very powerful to form spiral-arm substructures including gaseous spurs and giant clouds. The wiggle and Parker instabilities proposed for cloud formation appear to be suppressed by strong non-steady motions inherent in vertically-extended spiral shocks, suggesting that gravitational instability is a primary candidate for cloud formation.
\end{abstract}

Keywords. galaxies: ISM, instabilities, ISM: kinematics and dynamics, ISM: magnetic fields, method: numerical, MHD, stars: formation

\section{Introduction}

Most galactic star formation takes place in cold, giant molecular clouds (GMCs). Starforming GMCs distributed along spiral arms tend to appear in groups, forming giant molecular associations with mass $\sim 10^{7}-10^{8} M_{\odot}$ (e.g., Vogel et al. 1988; see also Tosaki et al. and Hitschfeld et al. in this volume for recent observational results). They are also closely associated with other spiral-arm substructures such as gaseous spurs (or feathers) and OB star complexes. Gaseous spurs are prominent in an HST image of M51 (Scoville \& Rector 2001) as short dust lanes jutting out almost perpendicularly from main spiral arms, and as warm dust filaments in a Spitzer Legacy image of M51 (Kennicutt 2004). The recent analyses of $H S T$ archive data by La Vigne et al. (2006) indicate that gaseous spurs are in fact very common in grand design spirals and coincide with the density peaks of molecular gas.

Many mechanisms have been proposed for the formation of giant clouds and spurs. These include collisional agglomeration of small clouds into large clouds, the Parker instability, and gravitational instability. Although the first, stochastic coagulation model has been successful in reproducing the observed GMC mass and velocity distributions (e.g., Das \& Jog 1996), the basic premise of this mechanism is doubtful because cloud collisions usually lead to disruption rather than merger (e.g., Kim et al. 1999). In addition, there is insufficient mass in small clouds to build a steady mass spectrum (e.g., Heyer \& Terebey 1998), and it takes too long time to achieve GMC masses (e.g., Blitz \& Shu 1980).

Parker (1966) showed that giant clouds can form at the magnetic valleys due to magnetic buoyancy force in a vertically stratified disk. Because the wavelengths and growth times of the most unstable Parker modes are comparable to observed GMC spacings and lifetime, the Parker instability has been favored for GMC formation (Blitz \& Shu 
1980). However, the results of nonlinear simulations suggest that the Parker alone cannot produce overdense structures like GMCs (e.g., Santillán et al. 2000; Kim et al. 2000). In fact, the Parker instability is self-limiting because of stabilizing magnetic tension forces. Neither galactic differential rotation (Kim, Ostriker, \& Stone 2003) nor spiral density waves (Kim \& Ostriker 2006a) helps the Parker instability much.

On the other hand, self-gravity is a long-range force and thus allows runaway growth of condensations. The observed typical mass and separation of giant clouds along spiral arms are consistent with the characteristic Jeans mass and length of galactic disks at large (e.g., Elmegreen 1987). Recent work of La Vigne et al. (2006) showed that the spacing of gaseous spurs in spiral galaxies is also in good agreement with that from gravitational instability operating inside spiral arms. In recent years, we have studied cloud formation via gravitational instability in a local patch of galactic disks (Kim \& Ostriker 2001, 2002, 2006a, 2006b; Kim, Ostriker, \& Stone 2002, 2003). Effects of magnetic fields, (both thin and thick) self-gravity, galactic differential rotation, spiral arms as well as an active stellar component have been included. In what follows, we highlight the differences among various self-gravitating instabilities and summarize the main results of our investigation. The interested reader is referred to the original work for more detailed discussion.

\section{Self-gravitating mechanisms}

\subsection{Axisymmetric instability}

Consider a self-gravitating, rotating, gaseous disk threaded by azimuthal magnetic fields. The disk has surface density $\Sigma$, angular velocity $\Omega$, sound speed $c_{s}$, Alfvén speed $v_{\mathrm{A}}$, and vertical scale height $H$. We concentrate on axisymmetric perturbations that do not rely on vertical motions. For local WKB modes for which the radial variations of all physical quantities (except $\Omega$ ) are unimportant, the dispersion relation for axisymmetric disturbances with wavenumber $k$ becomes

$$
\omega^{2}=\kappa^{2}+\left(c_{s}^{2}+v_{\mathrm{A}}^{2}\right) k^{2}-\frac{2 \pi G \Sigma|k|}{1+|k| H},
$$

where $\kappa \equiv\left(4 \Omega^{2}+d \Omega^{2} / d \ln R\right)^{1 / 2}$ is the epicyclic frequency (e.g., Kim, Ostriker, \& Stone 2002). Note that the denominator of the gravity term in equation (2.1) accounts approximately for the geometrical dilution of self-gravity due to finite disk thickness.

For infinitesimally thin, unmagnetized disks, one can show from equation (2.1) that disks become unstable only if $Q<1$, with the Toomre stability parameter $Q$ defined by

$$
Q \equiv \frac{\kappa c_{s}}{\pi G \Sigma}
$$

The range of unstable wavenumbers is $1-\left(1-Q^{2}\right)^{1 / 2}<2 k / k_{\mathrm{J}}<1+\left(1-Q^{2}\right)^{1 / 2}$, where the Jeans wavenumber $k_{\mathrm{J}} \equiv 2 \pi G \Sigma / c_{s}^{2}$. Sonic motions and Coriolis forces stabilize short and long wavelength perturbations, respectively. It should be noted that the usual critical value $Q_{c}=1$ applies only to axisymmetric instability in unmagnetized, razor-thin disks.

Clearly, the presence of magnetic fields plays a stabilizing role, decreasing the critical value to $Q_{c}=(1+1 / \beta)^{-1 / 2}$, with the plasma parameter $\beta \equiv c_{s}^{2} / v_{\mathrm{A}}^{2}$. Finite disk thickness decreases the critical value, as well. For isothermal, unmagnetized, self-gravitating disks, equation (2.1) yields $Q_{c}=0.65$. Galactic disks are further compressed by the external stellar gravity. In the solar neighborhood, the strength of the external gravity is comparable to the self-gravity at one scale height, and in this situation equation (2.1) gives $Q_{c} \sim 0.75,0.72$, and 0.57 for $\beta=\infty, 10$, and 1 cases, respectively (Kim, Ostriker, \& Stone 2002). 
On the other hand, the presence of a dynamically-active stellar disk helps destabilize the system. Jog \& Solomon (1984) and many other authors analyzed axisymmetric stability of two-component (gas + stars) disks, treating the stellar disk as an isothermal fluid. Using a collisionless description of the stars, Rafikov (2001) derived a dispersion relation for axisymmetric waves in the combined, razor-thin disks. Kim \& Ostriker (2006b) extended Rafikov's work to allow for the effect of finite disk thickness. When the stellar parameters are similar to the solar neighborhood conditions, they found $Q_{c}=1.27$ for (unrealistically) razor-thin disks, while $Q_{c}=0.67$ when both disks possess realistic scale heights, implying that the stabilizing effect of finite disk thickness is considerable. Although observed star formation thresholds at $Q_{\mathrm{th}} \sim 1.4$ have often been attributed to gravitational instability in two-component disks (e.g., Martin \& Kennicutt 2001), it should not be a consequence of axisymmetric gravitational instability.

\subsection{Swing amplification}

Since real perturbations are more likely non-axisymmetric, axisymmetric gravitational instability, albeit mathematically simple, would not be readily materialized in real disk galaxies. In more general, non-axisymmetric cases, perturbations are able to amplify either through swing amplification or magneto-Jeans instability.

Swing amplification arises due to the conspiracy among background shear, epicyclic shaking, and self-gravity (e.g., Toomre 1981). The kinematics of background shear causes the wavefronts of disturbances to rotate from leading to trailing, which occurs in the same sense as epicyclic motions. Consequently, fluid elements stay longer in wave crests, enhancing self-gravity and amplifying perturbations. Swing amplification is not a true instability but a transient mechanism, efficient only when disturbances are loosely wound. The density amplification factor is largest when the local shear rate $q \equiv-d \ln \Omega / d \ln R \sim$ 1 , a condition easily met at outer galactic disks, while tending to zero as $q$ decreases.

While swing amplification in the linear theory yields an amplification factor that is a continuous function of $Q$, numerical simulations show that it exhibits nonlinear $Q$ threshold behavior for gravitational runaway. That is, when $Q<Q_{c}$, swing amplification puts the system in a state where nonlinear interactions of swing-amplified filaments eventually cause bound cloud formation, while disks with $Q>Q_{c}$ remain stable with only mildly fluctuating density fields. Bound clouds that form in unstable models have a typical mass of a few $10^{7} M_{\odot}$, similar to the characteristic Jeans mass of initial disks. For razor-thin, gas-only disks, Kim \& Ostriker (2001) found $Q_{c} \sim 1.2-1.4$ for $\beta=1-\infty$, indicating that $Q_{c}$ for nonlinear swing amplifier is insensitive to the strength of azimuthal magnetic fields. Strong density fluctuations associated with magnetorotationally-driven turbulence in a vertically stratified disk increase $Q_{c}$ to 1.6 (Kim, Ostriker, \& Stone 2003).

Kim \& Ostriker (2006b) studied the effects on $Q_{c}$ of a live stellar component as well as finite disk thickness, by following the orbits of collisionless stars using a particle-mesh method. They found that the two effects nearly cancel each other, giving $Q_{c} \sim 1.4$ for the stellar parameters corresponding to the solar neighborhood. This $Q_{c}$ value is consistent with the recent results of Li, Mac Low \& Klessen (2005) for gravitational runaway in global models. The quantitative agreement between the numerically-obtained $Q_{c}$ and observationally determined thresholds for star formation suggests that nonlinear swing amplification of non-axisymmetric disturbances may be responsible for star formation boundaries of disk galaxies. 


\subsection{Magneto-Jeans instability}

Now, consider non-axisymmetric perturbations in disks with no (or weak) shear. The instantaneous dispersion relation then reads

$$
\omega^{4}-\left[\kappa^{2}+\left(c_{s}^{2}+v_{\mathrm{A}}^{2}\right) k^{2}-\frac{2 \pi G \Sigma|k|}{1+|k| H}\right] \omega^{2}+v_{\mathrm{A}}^{2} k_{y}^{2}\left(c_{s}^{2} k^{2}-\frac{2 \pi G \Sigma|k|}{1+|k| H}\right)=0,
$$

where $k^{2}=k_{x}^{2}+k_{y}^{2}$, with $k_{x}$ and $k_{y}$ denoting the perturbation wavenumbers in the radial and azimuthal directions, respectively (e.g., Lynden-Bell 1966; Kim, Ostriker, \& Stone 2002). Note that equation (2.3) recovers equation (2.1) when $k_{y}=0$. When $k_{y} \neq 0$, the instability criterion is the same as the usual two-dimensional Jeans condition (modified by thick-disk gravity) in the absence of rotation and magnetic fields; that is, magnetic fields removes the stabilizing effect of galactic rotation.

In a rotating disk, a hurdle to overcome for self-gravitating modes to grow is Coriolis forces that cause epicyclic gas motions. When perturbations are non-axisymmetric, azimuthal magnetic fields exert tension forces that resist epicyclic orbits across the field lines. The constraint of potential vorticity conservation does no longer hold, and a contracting region is able to grow. This destabilizing effect of magnetic tension on nonaxisymmetric perturbations is in sharp contrast to the stabilizing effect of magnetic pressure on axisymmetric modes. Since the presence of magnetic fields is essential for non-axisymmetric instability, we term this magneto-Jeans instability (MJI). The MJI grows rapidly at a rate of $\sim \Omega^{-1}$ and occurs under low shear conditions; when shear is strong, $k_{x}$ increases rapidly with time and the sonic term in equation (2.3) dominates eventually. The MJI may be responsible for star burst activity toward the galactic central parts where rotation curves are almost linearly rising. Also, a spatially varying sense of shear makes spiral arms ideal places for the operation of MJI to produce spiral-arm substructures, as discussed in the next section.

\section{Cloud formation inside spiral arms}

The presence of stellar spiral potential perturbations not only compresses the gas and magnetic fields into spiral shocks but also changes the velocity structure significantly, causing streaming motions near the spiral arms. For isothermal spiral arms, the conservation of potential vorticity requires the Toomre $Q$ parameter and the local shear rate $q$ to vary as

$$
Q=Q_{0}\left(\frac{\Sigma}{\Sigma_{0}}\right)^{-1 / 2}, \quad q=2-\left(2-q_{0}\right)\left(\frac{\Sigma}{\Sigma_{0}}\right)
$$

where $\Sigma_{0}$ is the mean density and $Q_{0}$ and $q_{0}$ refer to the respective values in the absence of the spiral arm forcing (e.g., Kim \& Ostriker 2002). For flat rotation with $q_{0}=1$, spiral arm regions with $\Sigma / \Sigma_{0}>2$ experience shear reversal and thus are not prone to swing amplification. With low surface density, on the other hand, interarm regions achieve stronger forward shear. This spatially varying (i.e., reversed shear followed by normal shear) sense of shear caused by spiral arms maintains the overall shear rate small. With high density, strong magnetic fields, and low net shear, therefore, spiral arm regions are favorable places for the development of MJI (e.g., Elmegreen 1987, 1994).

Numerical simulations of local spiral arms indeed show that spiral arms with sufficient peak density are stable to swing amplification but unstable to MJI. Figure 1 shows evolution of surface density and magnetic fields due to MJI in a two-dimensional thickdisk model presented in Kim \& Ostriker (2006a). As perturbations grow, gaseous spurs emerge nearly perpendicularly downstream from the spiral shock and become trailing in 
(a) $t=2.1 t_{\text {ort }}$

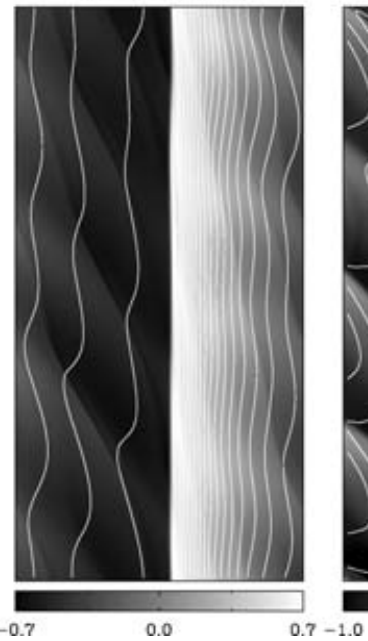

(b) $\mathrm{t}=2.5 \mathrm{t}_{\text {orb }}$

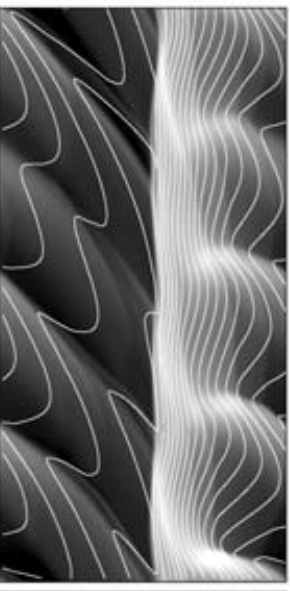

0.0 (c) $\mathrm{t}=3.2 \mathrm{t}_{\text {orb }}$

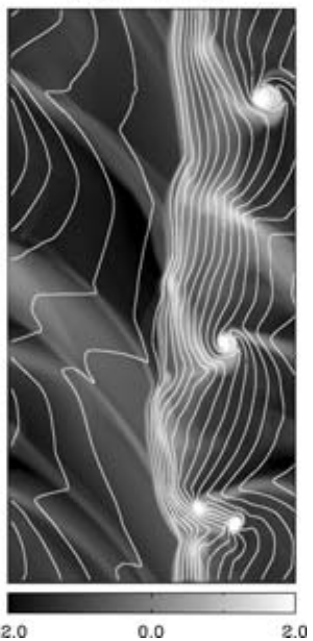

Figure 1. Snapshots of gas surface density (logarithmic gray scale) and magnetic field lines (solid curves) for a local two-dimensional spiral arm model with $Q=1.2, \beta=10$, and a spiral arm strength of $10 \%$. This model takes allowance for finite disk thickness in self-gravity.

the interarm regions (Fig. 1b), simply reflecting the kinematics of shearing and expanding background flows off the spiral arm. Observed spurs have similar shapes. In fully threedimensional disks, the mean separation of MJI-driven spurs is about 10 times the Jeans length at the arm peak, which is also consistent with observations (see La Vigne et al. 2006). When spurs grow and become sufficiently nonlinear, they experience fragmentation to form gravitationally bound condensations (Fig. 1c). These bound clouds have a mean mass of a few $10^{7} M_{\odot}$, are magnetically supercritical, and would evolve into arm and interarm HII regions. Although magnetic fields pinch inward within the spurs, Figure 1 shows that they almost parallel the spiral arms overall, demonstrating that the material in MJI is collected along the spiral arms.

Wada \& Koda (2004) showed that spiral shocks in two-dimensional disks (with the vertical dimension suppressed) are unstable to vorticity-generating wiggle instability. The nature of the wiggle instability is not well known, but numerical work suggests that it is potentially Kelvin-Helmholtz instabilities at a spiral shock; it needs spiral shocks to be quite strong; it requires neither magnetic fields nor self-gravity; and it appears to be suppressed by the equi-partition magnetic fields (Shetty \& Ostriker 2006). Most importantly, the wiggle instability is absent in full three-dimensional disks (Kim \& Ostriker 2006a). When the vertical dimension is explicitly included, spiral shocks exhibit vigorous non-steady motions and strong vertical shear (Kim, Kim, \& Ostriker 2006), preventing the growth of coherent vortical structure that is essential for the wiggle instability. These turbulent gas flows across spiral shocks in vertically extended disks appear to suppress the Parker instability, as well.

\section{Summary}

Recent numerical magnetohydrodynamic simulations have investigated giant cloud formation in the presence of self-gravity, magnetic fields, galactic differential rotation, dynamically active stars, and/or passive stellar density waves, all of which are crucial for galactic gas dynamics. These works have shown that self-gravity plays a fundamental role in the formation of giant clouds and spiral-arm substructures. When shear is strong, 
as in outer galaxies without strong spiral arms, swing amplification is subject to nonlinear threshold behavior such that disks with $Q<Q_{c}$ undergo runaway collapse to form bound clouds of a few $10^{7} M_{\odot}$, roughly the Jeans mass. For swing amplifier, the inclusion of an active stellar component nearly compensates for the stabilizing effect of finite disk thickness. For the parameters representing the solar neighborhood conditions, $Q_{c} \sim 1.4$, similar to the observationally-inferred thresholds for active star formation. When shear is weak, on the other hand, as in galactic central regions or inside spiral arms, gaseous spurs and giant clouds naturally form as a consequence of magneto-Jeans instability that critically relies on magnetic tension forces to resist the stabilizing Coriolis force of galaxy rotation. The MJI predicts a mean spur separation of 10 times the Jeans length at the arm peak and an average cloud mass of a few $10^{7} M_{\odot}$, consistent with the observed spur spacings and giant cloud masses near spiral arms. Non-steady flows associated with spiral shocks in vertically stratified disks stabilize the wiggle and Parker instabilities, making them unlikely mechanisms for giant cloud formation in real disk galaxies.

\section{Acknowledgements}

I gratefully acknowledge Eve Ostriker for her stimulating advice and constructive comments. This work is supported in part by Korea Science and Engineering Foundation (KOSEF) grant R01-2004-000-10490-0.

\section{References}

Blitz, L. \& Shu, F.H. 1980, ApJ 238, 148

Das, M. \& Jog, C.J. 1996 ApJ 462, 309

Elmegreen, B.G. 1987, ApJ 312, 626

Elmegreen, B.G. 1994, ApJ 433, 39

Heyer, M.H. \& Terebey, S. 1998, ApJ 502, 265

Jog, C.J. \& Solomon, P.M. 1984, ApJ 276, 114

Kennicutt, R.C. 2004, Spitzer press release at http://www.spitzer.caltech.edu/Media/ releases/ssc2004-19/ssc2004-19a.shtml

Kim, C.-G., Kim, W.-T. \& Ostriker, E.C. 2006, ApJ 649, L13

Kim, J., Franco, J., Hong, S.S., Santillán, A. \& Martos, M.A. 2000, ApJ 531, 873

Kim, W.-T. \& Ostriker, E.C. 2001, ApJ 559, 70

Kim, W.-T. \& Ostriker, E.C. 2002, ApJ 570, 132

Kim, W.-T. \& Ostriker, E.C. 2006a, ApJ 646, 213

Kim, W.-T. \& Ostriker, E.C. 2006b, ApJ submitted

Kim, W.-T., Ostriker, E.C. \& Stone, J.M. 2002, ApJ 581, 1080

Kim, W.-T., Ostriker, E.C. \& Stone, J.M. 2003, ApJ 599, 1157

Kim, W.-T., Hong, S.S., Yoon, S.-C., Lee, S.M. \& Kim, J. 1999, in Numerical Astrophysics eds. S.M. Miyama, K. Tomisaka, \& T. Hanawa (Boston: Kluwer), 111

La Vigne, M.A., Vogel, S.N. \& Ostriker, E.C. 2006, ApJ in press; astro-ph/0606761

Li, Y., Mac Low, M.M. \& Klessen, R.S. 2005, ApJ 620, L19

Lynden-Bell, D. 1966, Observatory 86, 57

Martin, C.L. \& Kennicutt, R.C. 2001, ApJ 555, 301

Parker, E.N. 1966, ApJ 145, 811

Rafikov, R.R. 2001, MNRAS 323, 445

Santillán, A., Kim, J., Franco, J., Martos, M., Hong, S.S. \& Ryu, D. 2000, ApJ 545, 353

Scoville, N. \& Rector T. 2001, HST press release at http://oposite.stsci.edu/pubinfo/PR/ 2001/10/index.html

Toomre, A. 1981, in: S.M. Fall \& D. Lynden-Bell (eds.), Structure and Evolution of Normal Galaxies (Cambridge: Cambridge Univ. Press), p. 111

Vogel, S.N., Kulkarni, S.R. \& Scoville, N.Z. 1988, Nature 334, 402

Wada, K. \& Koda, J. 2004, MNRAS 349, 270 


\section{Discussion}

HANASZ: (1.) I don't agree with your statement that the Parker instability is irrelevant. It may be indeed irrelevant if cosmic rays are neglected. However, if cosmic rays are taken into account, the Parker instability becomes very violent and its timescale is very short. (2.) Density condensations in the Parker instability are very small only in isothermal or adiabatic approximation. If realistic cooling and heating mechanisms are taken into account, then it appears that the combined action of Parker and thermal instabilities produced high density condensations, even in a thermally stable medium (see Kisinski \& Hanasz, MNRAS 2006).

KIM: Numerical studied (e.g., J. Kim et al. 2001) suggest that the Parker instability "alone" even with the effect of cosmic rays included cannot produce large density enhancement. It is basically because the Parker instability is not a runaway process, stabilized by magnetic tension forces. So cosmic rays are unlikely to play a major role in cloud formation inside spiral arms, although it may be important for turbulence generation. (2.) Then it will be very interesting to see how clouds for in magnetized spiral arms under realistic cooling and heating. Time scales for thermal and Parker instabilities are very different with the former longer than the latter by about two orders of magnitude. So, I wonder if the Parker instability grows in an inhomogeneous medium already produced by gas cooling and heating in a paper you mentioned. 